\title{
Individualization and Electrical Characterization of SiGe Nanowires
}

\author{
M. Monasterio ${ }^{1}$, A. Rodríguez ${ }^{1}$, T. Rodríguez ${ }^{1}$, C. Ballesteros ${ }^{2}$ \\ ${ }^{1}$ Tecnología Electrónica, Universidad Politécnica de Madrid, E.T.S.I.T., 28040 Madrid, Spain \\ ${ }^{2}$ Física, Universidad Carlos III, 28911 Leganés (Madrid), Spain
}

\begin{abstract}
SiGe nanowires of different $\mathrm{Ge}$ atomic fractions up to $15 \%$ were grown and ex-situ n-type doped by diffusion from a solid source in contact with the sample. The phenomenon of dielectrophoresis was used to locate single nanowires between pairs of electrodes in order to carry out electrical measurements. The measured resistance of the as-grown nanowires is very high, but it decreases more than three orders of magnitude upon doping, indicating that the doping procedure used has been effective.
\end{abstract}

\section{INTRODUCTION}

Nanowires (NWs) with tailored physical properties can be considered key components in flexible electronics, renewable energy technologies, biological applications and sensing. Integrating these nanostructures into the corresponding devices requires their individualization and placement into desired specific locations. SiGe NWs are interesting for electronic devices since their electronic properties can be tailored over a wide range [1]. Among the different methods considered for the manipulation of nanostructures, the one based on the electric field guided assembly in a fluid (dielectrophoresis) has been successfully used with carbon nanotubes [2], gold NWs [3] as well as InAs [4], ZnO [5], Si [6] and $\mathrm{SiGe} \mathrm{[7]} \mathrm{semiconductor} \mathrm{NWs,} \mathrm{which}$ have been precisely aligned onto predefined electrodes. This procedure is carried out at room temperature and is compatible with the established technologies for devices fabrication. The physical aspects of the phenomenon on which this technique is based have been explained in detail [8] and several authors have experimentally analyzed the influence on the obtained results of the multiple variables involved, like the shape and size of the electrodes, the dielectric constants and conductivities of the nanostructures and the surrounding medium, the frequency and amplitude of the electrical excitation, the viscosity of the solution and the concentration of NWs $[3,5,9]$. This work is focused on the growth, n-type doping, individualization by dielectrophoresis and electrical characterization of as-grown and ex-situ doped SiGe nanowires.

\section{EXPERIMENTAL}

The growth of SiGe NWs was carried out on Si wafers by the Vapor-Liquid-Solid method using a Low Pressure Chemical Vapor Deposition reactor and $\mathrm{Ga}-\mathrm{Au}$ as catalyst [10]. Most of the NWs are straight and cylindrical in shape, with diameters around $30-40 \mathrm{~nm}$ and lengths of 2 $-6 \mu \mathrm{m}$. Different Ge atomic fractions (from 0 to $\approx 15 \%$ ) were obtained by changing the flow ratio (R) of the $\mathrm{GeH}_{4}$ and $\mathrm{Si}_{2} \mathrm{H}_{6}$ precursor gases. Doping (n-type) of the NWs was carried out by 
diffusion using two approaches $[11,12]$. First, a spin-on glass containing $\mathbf{P}$ was deposited on the samples and densified, in such way that the NWs were encapsulated by the dopant source during the subsequent annealing step [13]. The oxide was finally removed by etching using BHF. In the second method, the spin-on glass was deposited onto a bare Si substrate which was then placed in contact with the NWs sample. The annealing process was carried out in both cases at $800{ }^{\circ} \mathrm{C}$ for $30 \mathrm{~min}$ in $\mathrm{N}_{2}$ atmosphere. These process parameters were selected after a set of simulations using the SSuprem3 Code [14] to ensure that the diffusion length is longer than the NW radius and after a set of experiments oriented to verify that the NWs withstand the thermal treatment without a noticeable degradation. In the first case, most of the NWs are removed during the oxide etching, even with very low etching rates, so this approach was discarded due to its low yield.

The devices consisted of arrays of pairs of electrodes located in front of each other at distances from 0.5 to $10 \mu \mathrm{m}$, with tapered and rectangular shapes and contact pads. The structures were fabricated on heavily doped $\mathrm{Si}$ wafers with an $\mathrm{Al}$ film evaporated on the bottom side to act as a third electrode and a $100 \mathrm{~nm}$ thick thermal $\mathrm{SiO}_{2}$ layer grown on the top one. The main electrodes were formed by e-beam lithography using PMMA on a Cr-Au bilayer deposited on top of the oxide followed by a lift-off process.

To select the conditions for the experiments to be carried out, the intensity of the dielectric force was calculated using the simplified model proposed in the literature for several liquids, conductivities of the NWs and excitation frequencies $[3,5]$. Based on these calculations, the use of methanol and an excitation frequency of around $1 \mathrm{kHz}$ could yield an attractive force. The NWs were therefore separated from the substrate by sonication of the as-grown samples and collected in a methanol bath. The electrical excitation was achieved in two configurations using a sine wave with a peak-to-peak voltage of up to $10 \mathrm{~V}$. In the first approach, capacitive coupling, the voltage was applied between the bottom contact of the sample and the central one of the top part. The voltages induced between couples of floating electrodes located in the surroundings are in the order of several $\mathrm{V}$ to some tenths of $\mathrm{V}$, depending on the distance to the center. The other approach consisted of applying the voltage to the central couple of electrodes of the top part. In this case the voltages measured in the couples of floating electrodes were smaller than in the previous one. A few droplets of methanol containing the NWs were deposited on the sample, covering the whole area where the electrodes are located. Although both excitation methods gave successful results, the second one shows a major drawback, since the voltage needs to be increased to induce sufficiently high voltages in the floating electrodes, causing a noticeable rounding of the tips of the excited device, making it useless for future experiments.

Dark field images taken using an optical microscope as well as Scanning Electron Microscopy (SEM) were used to verify the correct location of single NWs and appropriate alignment between the electrodes. I-V measurements in DC were performed in a two-wire configuration using Keithley instruments and with applied voltages of up to $8 \mathrm{~V}$.

\section{RESULTS}

\section{NWs placement and shape of the electrodes}

Figure 1 shows the results of some of the alignment experiments carried out using SiGe NWs with $\approx 15 \%$ Ge and an electrical excitation of $6 \mathrm{~V}$ (peak-to-peak) and $1 \mathrm{kHz}$. 

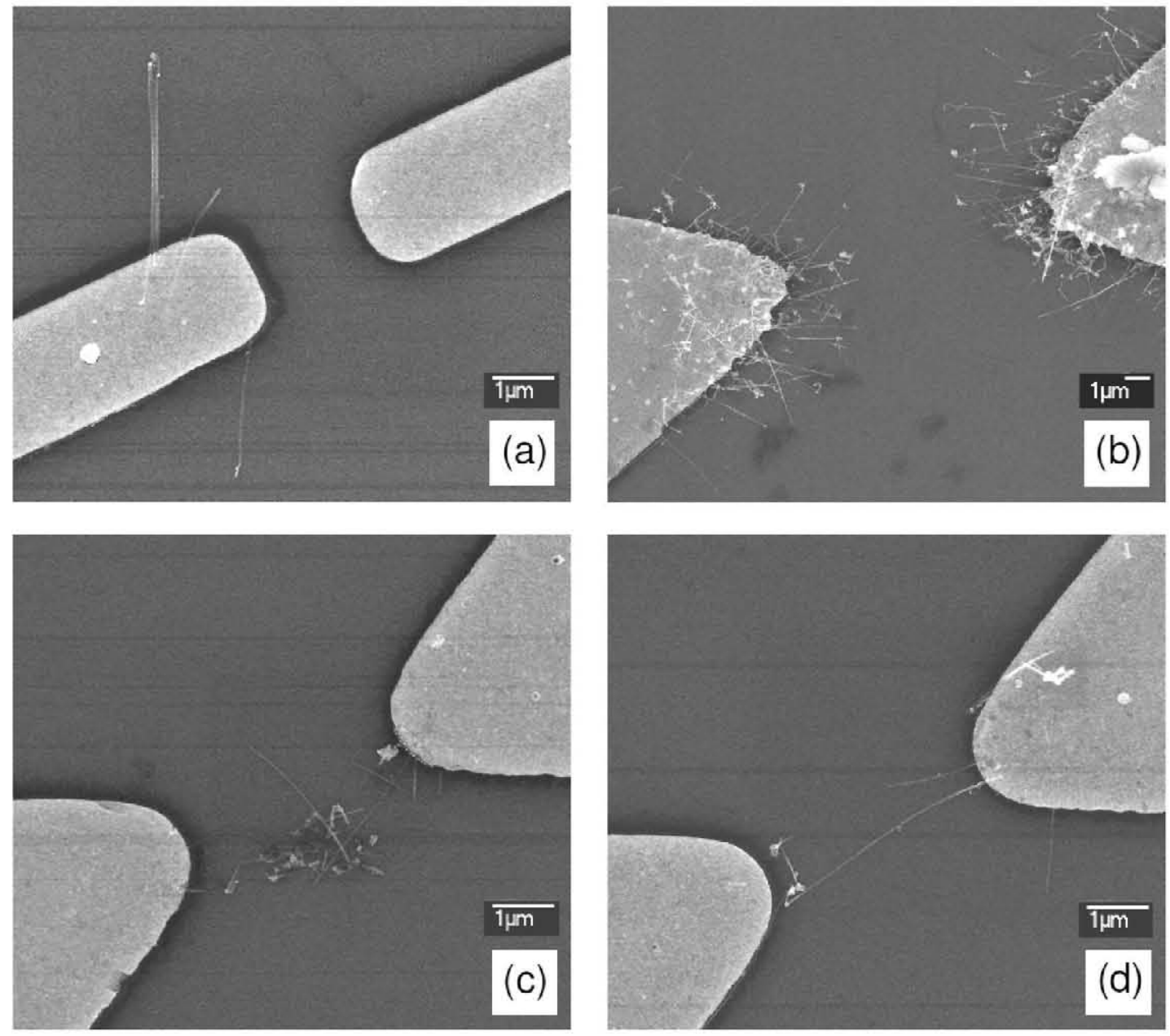

Figure 1. SEM images of different pairs of electrodes and NWs after dielectrophoresis experiments, all of them carried out using SiGe NWs with $15 \% \mathrm{Ge}$, and an electrical excitation of $6 \mathrm{~V}$ (peak-to-peak) and $1 \mathrm{kHz}$. The cases of rectangular electrodes (a), tapered electrodes with a gap much larger than the NW length (b, c) and tapered electrodes with a gap of the same order than the NW length (d) are shown.

The observations are in agreement with computer simulations carried out previously and reported in the literature [8]. If rectangular-shaped electrodes are used, see Figure 1 (a), the NWs appear to be always aligned perpendicular to the electrodes, instead of parallel to them. If the distance between the electrodes is smaller than the NW length, the short axis of the NW is the one that could be aligned with the electric field, in such way that the NW is placed orthogonal to the electrodes axis as it is observed (even though the gap is not much smaller than the NWs length). Figure 1 (b) shows the results of the experiment carried out in the same conditions using tapered electrodes several micrometers apart (the gap is several times the average NW length). The NWs are located delineating the field lines. Since the field distribution is not significantly perturbed by the incoming small NWs, more and more NWs are attracted and aligned. Figure 1 (c) shows another result obtained when the gap between the tapered electrodes is much larger then the NW length. In this case, some NWs are attracted towards the tip and oriented along the direction of the electric field. An intense and non uniform electric field appears at the ends of the aligned NW, which is much more tapered than the electrode, so other NWs experience this field and are also attracted to form local bundles. These bundles finally fill the area between the 
electrodes and bridge them. Figure 1 (d) shows a single NW of the same type aligned between two electrodes which gap matches almost exactly the NW length. In this case, it is usual that no more NWs are attracted to the area since the electric field is strongly perturbed by the presence of the first aligned NW $[2,8]$.

\section{Alignment of single of NWs}

Figure 2 shows dark field and SEM images of the results of some individualization events using $\mathrm{Si}$ and $\mathrm{SiGe}$ NWs of different compositions. Optical microscopy appears as a remarkably useful technique for a fast and in-situ monitoring of the process, prior to the use of ex-situ techniques like SEM to confirm that the NW is in contact with both electrodes. In the conditions described above, several (2-4) single NWs were usually located between couples of electrodes in each run when the sample with the tapered electrodes located at a distance matching the NW length was used. While bundles of NWs tend to accumulate near the center of the array, the successful events took place usually in electrodes located at a certain distance from it.
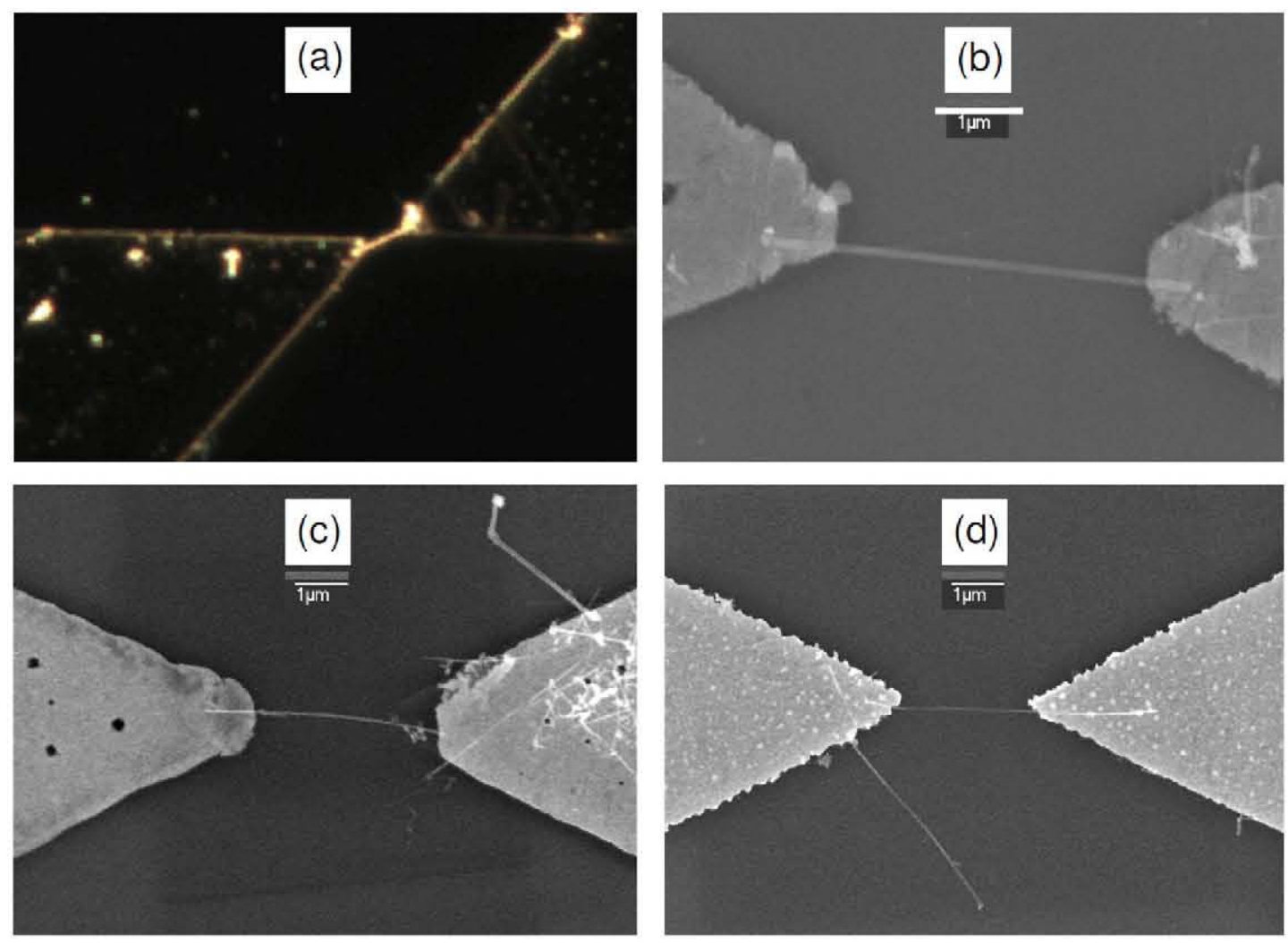

Figure 2. (a) Image of a Si NW ( $\mathrm{R}=0$, pure $\mathrm{Si}$ ) located between two tapered electrodes taken with an optical microscope (500×) in dark field mode. (b) SEM image of the same Si NW. (c) SEM image of a SiGe NW (R=1, $10 \% \mathrm{Ge})$. (d) SEM image of a SiGe NW $(\mathrm{R}=1.5,15 \% \mathrm{Ge})$.

\section{$\underline{\text { I-V Measurements }}$}

Figures 3 and 4 shows the I-V curves obtained in several as-grown and n-type doped SiGe NWs of different compositions. The same measurement has been carried out using a device 
formed by a couple of electrodes without NW for comparison. The results show that the resistance of the $\mathrm{Si}$ NW is so high that the I-V curve is indistinguishable from the one of the device with no NW. As the Ge content in the NW increases, enhanced conduction through the NWs is observed. These measurements are noisy, due to the low values of the current measured, and therefore several scans through the whole voltage range were carried out and averaged. Different shapes were observed in the I-V curves which point to slightly rectifier or nearly ohmic behaviors, depending on the nature of the nanowire-electrodes contacts. Neglecting the nonlinearity of the curves and taking into account the presence of the device in parallel, the obtained values of the resistance are around $90 \mathrm{G} \Omega$ and $30 \mathrm{G} \Omega$ for the NWs with $5 \%$ and $10 \%$ Ge respectively. The devices with the aligned NWs were not annealed, so these values may be significantly influenced by the contribution of the contacts [15]. Attributing all the measured resistance to the NW, the maximum value of the resistivity of the as-grown material is around $3000 \Omega \cdot \mathrm{cm}$ for the NW with $5 \% \mathrm{Ge}$ and $1000 \Omega \cdot \mathrm{cm}$ for the one with $10 \% \mathrm{Ge}$. These values are higher than the one reported for as-grown pure Si NWs, $390 \Omega \cdot \mathrm{cm}$, but these NWs were found to be p-type although they were not intentionally doped during their growth [16].

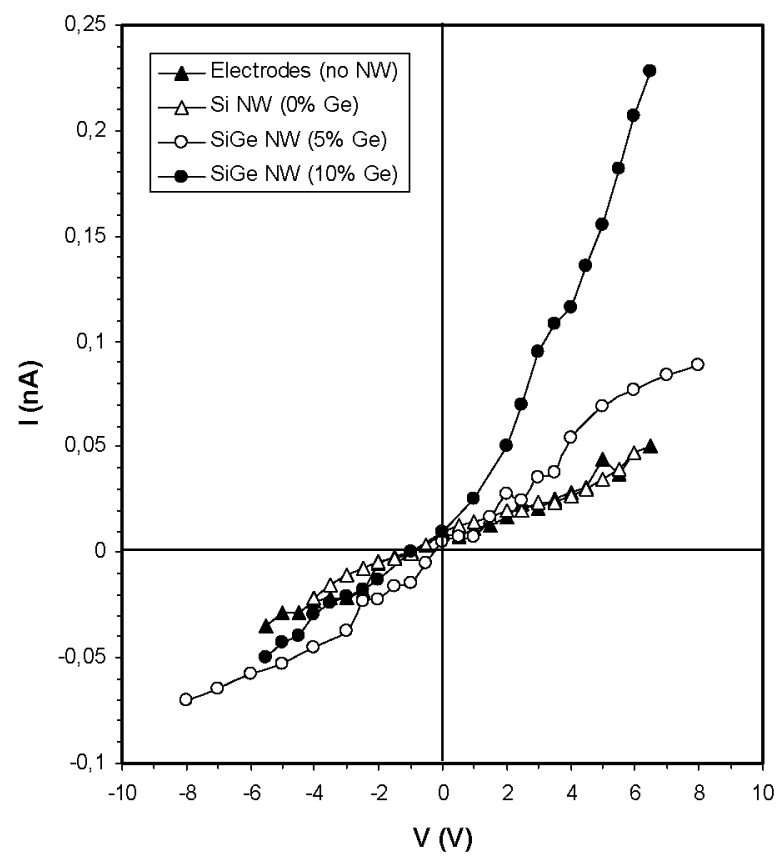

Figure 3. I-V measurements carried out in undoped SiGe NWs of different compositions.

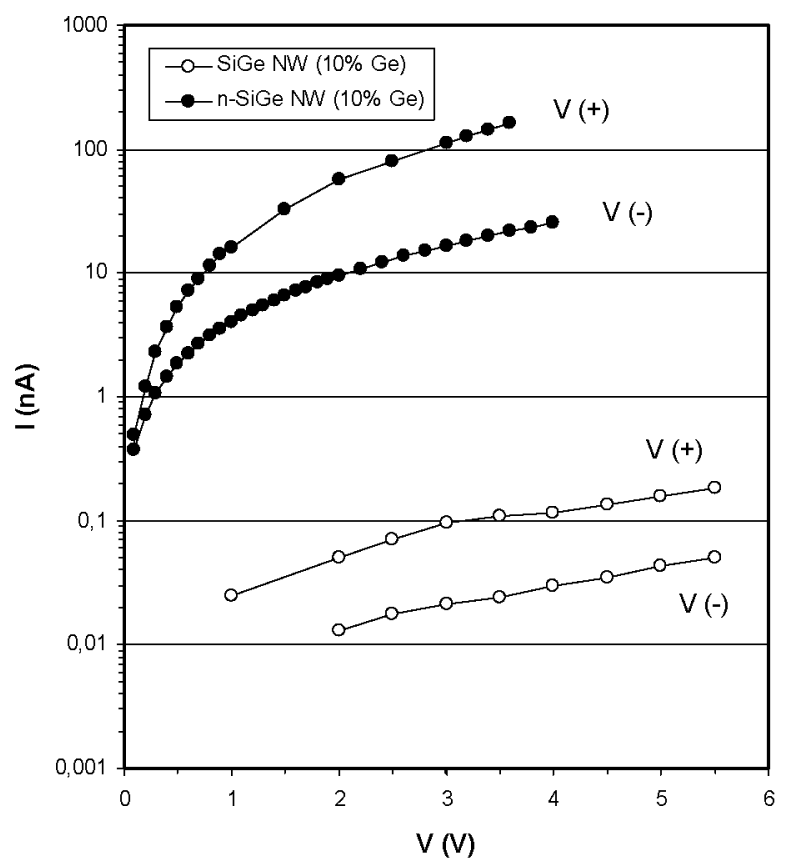

Figure 4. I-V measurements carried out in undoped and n-type doped SiGe NWs $(\approx 10 \% \mathrm{Ge})$.

The shape of the I-V curve of the doped NW with $10 \% \mathrm{Ge}$ also points to a rectifier-like behavior. The values of the current are three orders of magnitude higher than in the as-grown NWs, thus confirming the effectiveness of the doping procedure used. Again, attributing all the series resistance derived from the curve to the NW, a maximum value of the resistivity of the doped SiGe of $0.5 \Omega \cdot \mathrm{cm}$ is obtained. This value is consistent with the ones reported for $\mathrm{n}$ - and $\mathrm{p}$ doped pure Si NWs, which range from $260 \Omega \cdot \mathrm{cm}$ to $6.9 \times 10^{-3} \Omega \cdot \mathrm{cm}$ depending on the doping level. 


\section{SUMMARY}

SiGe NWs grown by the VLS method both as-grown as well as ex-situ n-type doped by diffusion have been located between two electrodes by dielectrophoresis. Using excitation by capacitive coupling, tapered electrodes located at a distance matching the NW length and methanol as fluid medium, several successful alignment events were obtained in each run. Darkfield optical microscopy has been shown to be very useful to get a fast and in-situ diagnostic of the success of the alignment experiments, prior to the use of ex-situ techniques like SEM.

$\mathrm{I}-\mathrm{V}$ measurements in as-grown NWs yield values of the resistance of $90 \mathrm{G} \Omega$ and $30 \mathrm{G} \Omega$ for NWs with Ge atomic fractions of 5\% and 10\% respectively. Doping of the NWs was achieved by diffusion using a solid source located next to the sample during the drive-in process. The measured resistance of a NW with a Ge atomic fraction of $10 \%$ after an annealing step at $800{ }^{\circ} \mathrm{C}$ for $30 \mathrm{~min}$. decreases more than three orders of magnitude, thus showing that the doping method is effective. Further work is being carried out to achieve p-type doping of the NWs and to minimize the influence of the contacts on the results.

\section{ACKNOWLEDGMENTS}

This work was funded by the Spanish Government (Grant: MAT2010-20441-C02-02).

\section{REFERENCES}

1. X. Zhang, K. K. Lew, P. Nimmatori, J. Redwing, E.C. Dickey; Nano Lett. 7, 3241 (2007).

2. A. Vijayaraghavan, S. Blatt, D. Weissenberger, M. Oron-Carl, F. Hennrich, D. Gerthsen, H. Hahn, R. Krupke. Nano Lett. 7, 1556 (2007)

3. J. J. Boote, S. D. Evans. Nanotechnology 16, 1500 (2005).

4. S. Raychaudhuri, S. Dayeh, D. Wang, T.Yu. Nano Lett. 9, 2260 (2009).

5. K. Jiang, W. Liu, L. Wan, J. Zhang. Sensors and Actuators B 134, 79 (2008).

6. M. Marczak, D. Hourlier, T. Mélin, L. Adamowicz, H. Diesinger. Appl. Phys. Lett. 96, $233502(2010)$.

7. C. Qi, G. Goncher, R. Solanki, J. Jordan. Nanotechnology 18, 075302 (2007).

8. Y. Liu, J.-H. Chung, W. K. Liu, R. S. Ruoff. J. Phys. Chem. B 110, 14098 (2006).

9. A. W. Maijenburg, M. G. Maas, E. J. B. Rodijk, W. Ahmed, E. S. Kooij. E. T. Carlen, D. H. A. Blank, J. E. ten Elshof. Journal of Colloid and Interface Science 355, 486 (2011).

10. M. Monasterio, A. Rodríguez, T. Rodríguez, C. Ballesteros. This Symposium.

11. K. E. Moselund, H. Ghoneim, H. Schmid, M. T. Björk, E. Lörtscher, S. Karg, G. Signorello, D. Webb, M. Tschudy, R. Beyeler, H. Riel. Nanotechnology 21, 435202 (2010).

12. S. Ingole, P. Aella, P. Manandhar, S. B. Chikkannanavar, E. A. Akhadov, D. J. Smith, S. T. Picraux. J. Appl. Phys. 103, 104302 (2008).

13. Desert Silicon, LLC. www.desertsilicon.com.

14. SSuprem3 1-D Process Simulation. www.silvaco.com.

15. W. Lu, C. M. Lieber. J. Phys. D: Appl. Phys. 39, R387 (2006).

16. Y. Cui, X. Duan, J. Hu, C. M. Lieber. J. Phys. Chem. B 104, 5214 (2000). 